\title{
Differential Cross Section Results from NuTeV
}

D. Naples* and J. McDonald

Department of Physics, University of Pittsburgh, PA E-mail: haples@phyast.pitt.edü

T. Adams ${ }^{4}$, A. Alton ${ }^{4}$, S. Avvakumov ${ }^{8}$, L. de Barbaro ${ }^{5}$, P. de Barbaro ${ }^{8}$,

R. H. Bernstein ${ }^{3}$, A. Bodek ${ }^{8}$, T. Bolton ${ }^{4}$, J. Brau ${ }^{6}$, D. Buchholz ${ }^{5}$ H. Budd ${ }^{8}$,

L. Bugel ${ }^{3}$, J. Conrad ${ }^{1}$, R. B. Drucker ${ }^{6}$, B. T. Fleming ${ }^{1}$, J. Formaggio ${ }^{1}$, R. Frey ${ }^{6}$,

J. Goldman ${ }^{4}$, M. Goncharov ${ }^{4}$, D. A. Harris ${ }^{8}$, J. H. Kim ${ }^{1}$, S. Koutsoliotas ${ }^{1}$,

R. A. Johnson ${ }^{2}$, M. J. Lamm ${ }^{3}$, W. Marsh ${ }^{3}$, D. Mason ${ }^{6}$, K. S. McFarland ${ }^{8}$,

C. McNulty ${ }^{1}$, P. Nienaber ${ }^{3}$, V. Radescu ${ }^{7}$, A. Romosan ${ }^{1}$,W. K. Sakumoto ${ }^{8}$,

H. Schellman ${ }^{5}$, M. H. Shaevitz ${ }^{1}$, P. Spentzouris ${ }^{1}$, E. G. Stern ${ }^{1}$, N. Suwonjandee ${ }^{2}$,

N. Tobien ${ }^{3}$, M. Tzanov ${ }^{7}$, A. Vaitaitis ${ }^{1}$, M. Vakili ${ }^{2}$, U. K. Yang ${ }^{8}$, J. $\mathbf{Y u}^{3}$, G. P. Zeller ${ }^{5}$,

E. D. Zimmerman ${ }^{1}$

${ }^{1}$ Columbia University, New York, NY, ${ }^{2}$ University of Cincinnati, Cincinnati, OH,

${ }^{3}$ Fermi National Accelerator Laboratory, Batavia, IL, ${ }^{4}$ Kansas State University, Manhattan, KS, ${ }^{5}$ Northwestern University, Evanston, $I L,{ }^{6}$ University of Oregon, Eugene, OR, ${ }^{7}$ University of Pittsburgh, Pittsburgh, PA, ${ }^{8}$ University of Rochester, Rochester, NY.

ABstract: Preliminary results for the neutrino-nucleon differential cross section from the $\mathrm{NuTeV}$ experiment are presented. Extraction of the structure functions $F_{2}$ and $R$ are discussed.

\section{Introduction}

Neutrino scattering offers a unique and complimentary probe of nucleon structure and QCD. The NuTeV experiment is a second generation neutrino deep inelastic scattering experiment using seperate high purity neutrino and antineutrino beams at Fermilab. $\mathrm{NuTeV}$ has potential to improve understanding of systematic uncertainties using its precision calibration beam designed to reduce the uncertainty of the absolute muon and hadron energy scale [i]. $6 \times 10^{5}$ neutrino $\left(\nu_{\mu}\right)$ and $3 \times 10^{5}$ anti-neutrino $\left(\bar{\nu}_{\mu}\right)$ charged-current interactions were observed in the $\mathrm{NuTeV}$ data taking run.

${ }^{*}$ Speaker. 
$\nu-\mathrm{Fe}$ structure functions are extracted by fitting the sum of neutrino and antineutrino differential cross sections. In terms of $\epsilon$ (the polarization of the virtual $W$ ) the sum of the anti-neutrino and neutrino differential cross sections can be written:

$$
F(\epsilon)=\frac{\pi(1-\epsilon)}{y^{2} G_{F}^{2} M E_{\nu}}\left(\frac{d^{2} \sigma^{\nu}}{d x d y}+\frac{d^{2} \sigma^{\bar{\nu}}}{d x d y}\right)=2 x F_{1}\left[1+\epsilon R\left(x, Q^{2}\right)\right]+g(y) \Delta x F_{3} .
$$

Where $G_{F}$ is the Fermi weak coupling constant, $\mathrm{M}$ is the mass of the proton, $E_{\nu}$ is the incident neutrino energy, and $y$, the inelasticity, is the fraction of energy transferred to the hadronic system. The $y$-dependence of the differential cross section is contained in the two functions, $\epsilon=\frac{2(1-y)-M_{p} x y / E}{1+(1-y)^{2}+M_{p} x y / E}$ and $g(y)=\frac{y(1-y / 2)}{1+(1-y)^{2}}$. A fit to the $\epsilon$ dependence of the cross section at fixed Bjorken $x$ and $Q^{2}$ can be used to determine the structure functions. $F_{2}$ is given by $F_{2}=2 x F_{1}\left(1+R\left(x, Q^{2}\right)\right) . R=\frac{\sigma_{L}}{\sigma_{T}}$ is the ratio of cross sections of longitudinally to transversely polarized $W$-bosons and $\Delta x F_{3}=x\left(F_{3}^{\nu}-F_{3}^{\bar{\nu}}\right)$ in leading order is the contribution for scattering off of heavy quarks given by $\approx 4 x(s-c)$. Correlation in the terms $\Delta x F_{3}=x\left(F_{3}^{\nu}-F_{3}^{\bar{\nu}}\right)$ and $R$ in fits over a limited range in $y$ requires input from higher-order QCD calculations or data for one of these terms.

\section{Extracting the Differential Cross Sections}

The differential cross section is determined from the differential number of events $\frac{d^{2} N}{d x d y}$ and the normalized flux $L(E)$ at a given neutrino energy,

$$
\frac{d^{2} \sigma^{\nu(\bar{\nu})}}{d x d y}=\frac{1}{L(E)} \frac{d^{2} N^{\nu(\bar{\nu})}}{d x d y} .
$$

In Eq. $1 \overline{1}-2$, , the kinematic variables $x$ and $y$ represent the Bjorken scaling variable and the inelasticity. The NuTeV kinematic range extends from about $10^{-3}$ to 0.95 in $x$ and from 0.05 to 0.95 in $y$; the energy reach is from $30 \mathrm{GeV}$ to about $400 \mathrm{GeV}$.

The differential number of events is determined from a sample of events which pass charged current quality cuts, which demand event containment, a minimum hadronic energy $(\nu)$ of $10 \mathrm{GeV}$, a momentum analyzed muon, and a minimum $Q^{2}$. The selected events are binned in $x, y$, and $E$; are corrected for detector effects; and are acceptance corrected using a fast detector simulation. The binning is chosen to approximately reflect the detector resolution.

A nearly orthogonal sample of events is selected to determine the flux. These events must pass a maximum hadronic energy cut of $20 \mathrm{GeV}$. The flux is solely a function of the neutrino energy and is determined by expanding the differential cross section in terms of $\frac{\nu}{E}$. The flux is proportional to the number of events in this sample, up to corrections of order $\frac{\nu}{E}$. Corrections up to $\left(\frac{\nu}{E}\right)^{2}$ are obtained and applied to determine the relative flux as a function of energy. The flux normalization is obtained by normalizing our total neutrino cross section to the world average value of $\frac{\sigma_{W O R L D}^{\nu}}{E}=0.677 \times 10^{-38} \mathrm{~cm}^{2} / \mathrm{GeV}$

The fast detector simulation, which takes into account acceptance and resolution effects, includes an empirically determined set of parton distribution functions with QCD evolution. [2] The parton distribution functions are determined by fitting the extracted 

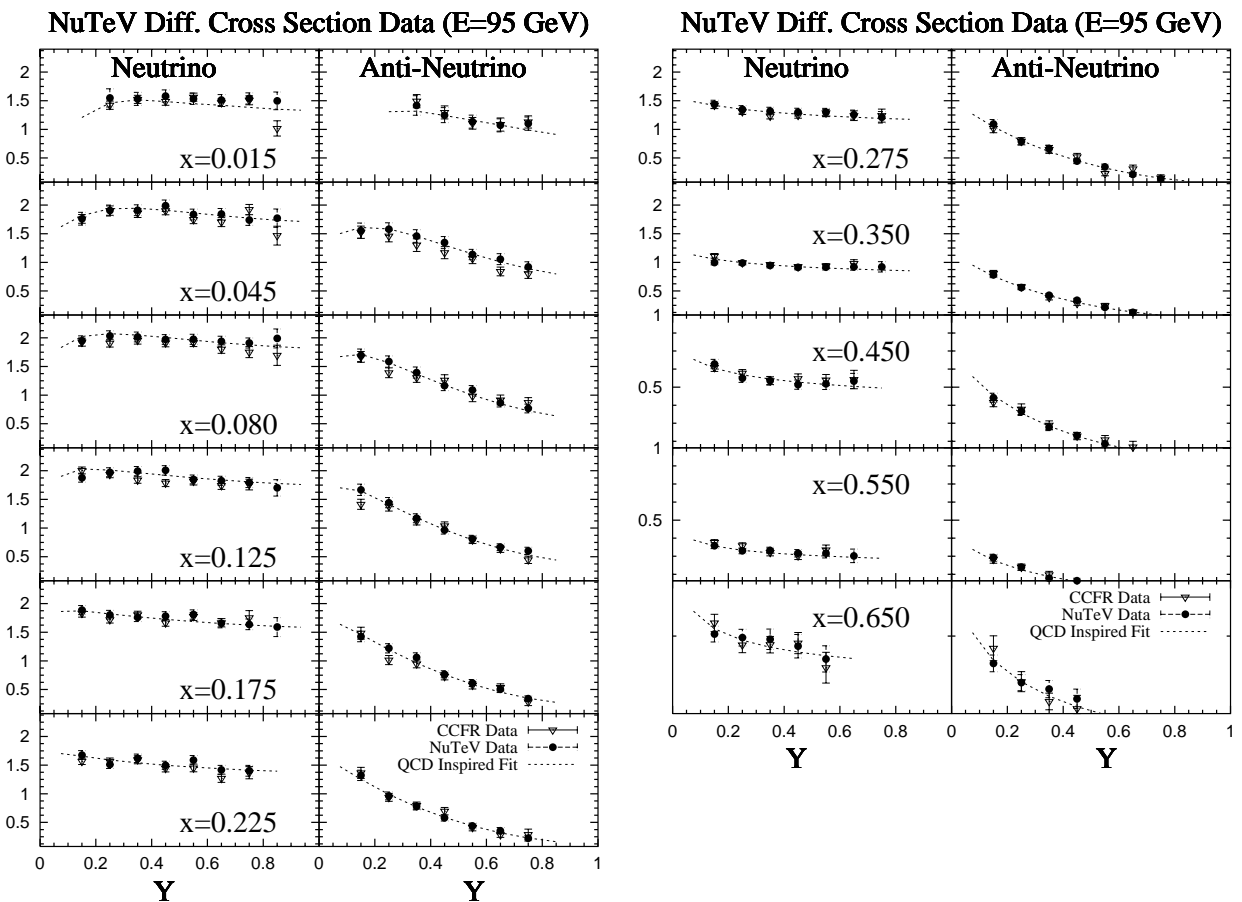

Figure 1: Neutrino and anti-neutrino (side by side) differential cross section from $\mathrm{NuTeV}$ (closed circles) and CCFR (open triangles) as a function of $y$ with the QCD inspired fit to the NuTeV data. Only statistical errors are shown. The differential cross section shown is in units of $G_{F}^{2} M / E_{\nu}$. The dashed line is the empirical fit to the differential cross section used to generate smearing and acceptance corrections.

differential cross section. The fitted parton distributions are used to determine acceptance corrections for both the DIS and flux sample. The procedure is then iterated. As a typical example, the differential cross section at $E=95 \mathrm{GeV}$ is shown in Fig. ${ }_{i}^{1} \mathrm{NuTeV}$ is found to be in good agreement with CCFR. [3] $\left[\begin{array}{l}3 \\ 1\end{array}\right]$

\section{Structure Functions}

$F_{2}, R$, and $\Delta x F_{3}$ cannot be simultaneously fit due to limited range in $y$ and strong correlations among the parameters. To performs fits to Eq. either $R\left(x, Q^{2}\right)$ or $\Delta x F_{3}$ must be used as input. NLO QCD models of $\Delta x F_{3}$ from ACOT fixed flavor scheme (ACOT-FFS) using GRV parton distributions; ACOT variable flavor scheme using CTEQ4HQ (ACOT-VFS); and Thorne-Roberts variable flavor scheme with

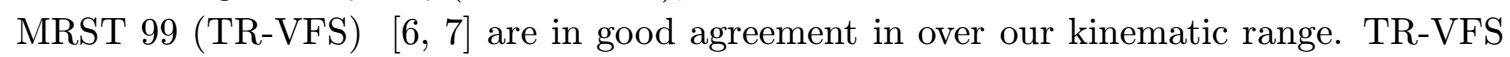
$\left[{ }^{\prime} \overline{6}_{1}^{\prime}, \overline{7}_{1}\right]$ is used as input for $\Delta x F_{3}$. Furthermore, $\Delta x F_{3}$ is small for $x>0.1 . R\left(x, Q^{2}\right)$ is well determined above $x>0.1$ from data [5] is used to determine $2 x F_{1}$ and $R$ for the $x<0.1$ range and a one parameter fit determines $2 x F_{1}$ for $x>0.1$.

Figure $i_{-1}^{1}$ shows an example fit and the results for $F_{2}\left(x, Q^{2}\right)$. The curve is the TR-NLO VFS calculation [i, $[\bar{a}]$. A reliable measurement of $R$ cannot be determined with incomplete 


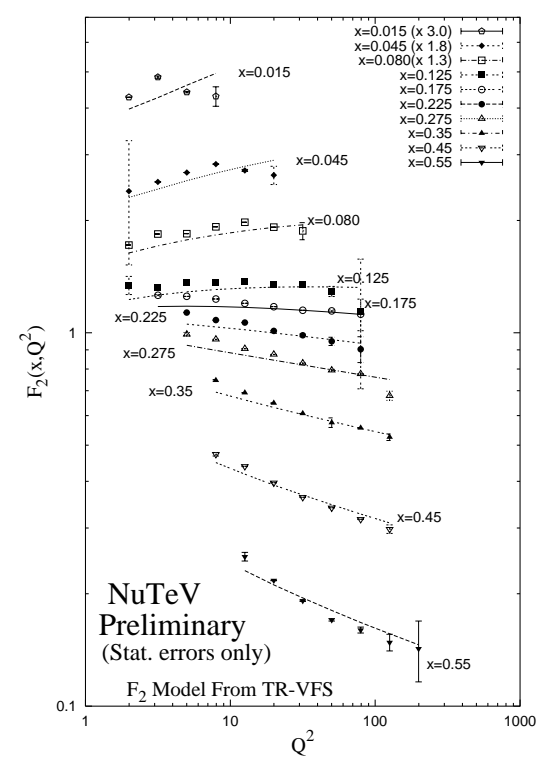

Figure 2: The extracted values of $F_{2}\left(x, Q^{2}\right)$ from $\mathrm{NuTeV}$ (statistical errors only) and a curve showing the theoretical prediction for $F_{2}$ as a function of $Q^{2}$ using TR-VFS.
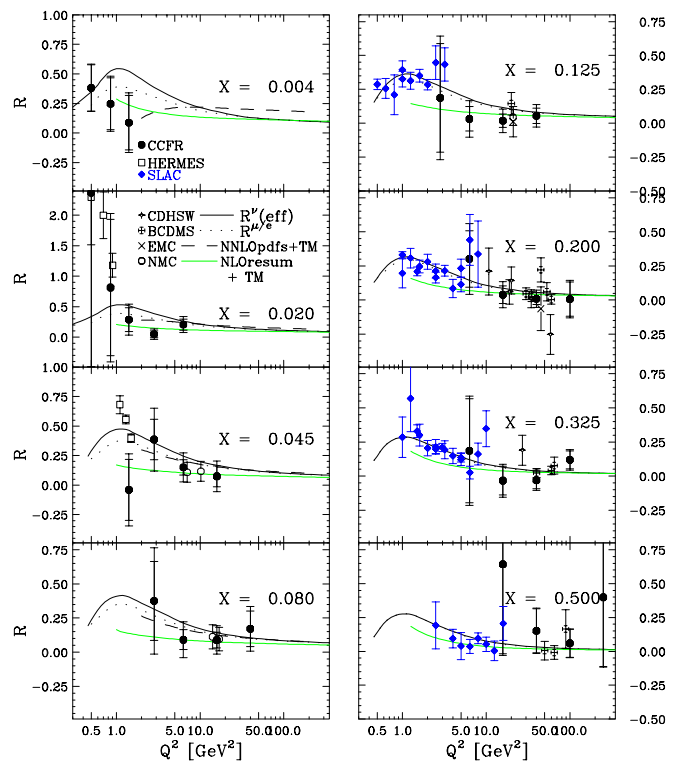

Figure 3: CCFR measurement of $R$.

knowledge of systematic uncertainties.

CCFR used the same method to make the first detemination of $R$ in the low $x$ region "8in]. Figure 1 i shows the CCFR determination and the world data on $R$. Our data is in agreement with $R_{\text {world }}$ and QCD models.

\section{Conclusions and Prospects}

Preliminary physics results of the $\mathrm{NuTeV}$ differential cross section results and structure 
functions have been extracted. The prospects for improving upon the current preliminary measurement and the CCFR measurement rely on the improved calibration for $\mathrm{NuTeV}$ and the extended kinematic range.

A unique feature of the $\mathrm{NuTeV}$ experiment is the sign-selected beam which allows the experiment to unambigiously assign the neutrino sign in a given event. The sign-selected beam allows one to expand the usual charge-current data sample of toroid-analyzed events to include those events which can be reconstructed using exclusively information from their energy deposition in the target calorimeter. These high-y data sets contribute a nearly independent set of data to the data sample presented here. This is where the sensitivity to $R\left(x, Q^{2}\right)$ and $\Delta x F_{3}$ is the greatest. The extension of the y-range in $\mathrm{NuTeV}$ may better constraint the fits and improve the determination of structure functions from $\mathrm{NuTeV}$.

\section{References}

[1] D. A. Harris, NIM A 447, 377 (2000).

[2] A. J. Buras and K. L. F. Gaemers, Nucl. Phys. B 132, 2109 (1978).

[3] U. K. Yang, Phys. Rev. Lett. 86, 2742 (2001).

[4] A. D. Martin, R. G. Roberts, W. J. Stirling, and R. S. Thorne, Eur. Phys. J. C 18, 117 (2000).

[5] L. W. Whitlow ,Phys. Lett. B 250, 193 (1990).

[6] R. S. Thorne and R. G. Roberts, Phys. Lett. B 421, 303 (1998).

[7] M. Aivazis, J. Collins, F. Olness, and W. K. Tung, Phys. Rev D 50, 3102 (1994).

[8] U. K. Yang, to be published in Phys. Rev. Lett., hep-ex/0104040. 